\title{
Interação Mãe-Bebê: A Relação Entre o Processo de Vinculação e as Práticas Parentais
}

\author{
Suzana Saab de Souza Zarske \\ Veronica Aparecida Pereira \\ Olga Maria Piazentin Rolim Rodrigues
}

\section{RESUMO}

Buscou-se descrever as práticas parentais de mães de bebês aos três meses e investigar o seu efeito sobre a interação mãe-bebê. Participaram do estudo, 39 díades mães-bebê. As mães responderam a um Inventário de Estilos Parentais para Pais e Mães de Bebês (IEPMB). As díades foram filmadas em condição estruturada (Face-to-Face Still Face - FFSF). As práticas parentais identificadas a partir do IEPMB foram classificadas como Positivas ou Negativas, distribuídas entre os níveis Baixo, Médio e Alto. Os comportamentos observados durante o FFSF foram categorizados em Orientação Social Positiva (OSP), negativa (OSN) (para bebês e mães) e Autorregulação do bebê. Houve predominância de práticas parentais positivas, com efeito da Prática Positiva Alta indicando maior ocorrência de autorregulação dos bebês durante o $2^{\circ}$ episódio. A interação dos fatores indicou efeito sobre a OSP dos bebês e suas mães, no $3^{\circ}$ episódio, com melhores resultados para mães com Prática Positiva Média e Negativa baixa. Os resultados podem subsidiar programas de intervenção na primeira infância.

Palavras-chave: práticas parentais; bebês; interação mãe-bebê.

\section{ABSTRACT \\ Mother-Baby Interaction: The Relationship Between the Bonding Process and Parentenal Pratictices}

This research aims to practices the parenting practices of three-month-old babies' mothers and to investigate its effect on mother-baby interaction. Thirty-nine mother-baby dyads participated in the study. The mothers responded to an Inventory of Parenting Styles for Fathers and Mothers Babies (IEPMB). The dyads were filmed in a structured condition (Face-to-Face Still Face - FFSF). The Parental practices identified from the IEPMB were classified as Positive or Negative, distributed among the Low, Medium and High levels. The Interactive behaviors were categorized into Positive Social Orientation (OSP) or Negative (OSN) (for babies and mothers) and baby Self-Regulation. There was a predominance of positive parenting practices, with the effect of High Positive Practice indicating a higher occurrence of babies' self-regulation during the 2 nd episode. The interaction of the factors indicated an effect on the OSP of babies and their mothers in the 3rd episode, with better results for mothers with Medium Positive and Low Negative Practices. The results can subsidize early childhood intervention programs.

Keywords: parenting practices; babies; mother-baby interaction

A relação mãe-criança nos primeiros anos de vida tem sido objeto de investigação de muitos estudos psicológicos na área do desenvolvimento humano (Alvarenga \& Cerezo, 2013; Chiodelli et al., 2020; Fuertes et al., 2011; Tronick et al., 1978). Isso se deve ao impacto que a relação e o vínculo afetivo estabelecidos entre a díade têm sobre o desenvolvimento do indivíduo na infância, estendendo-se às outras etapas do desenvolvimento (Arpini et al., 2016; Nunes et al., 2015). A interação mãe-bebê é uma oportunidade para que a mãe possa desenvolver no-

\section{Sobre os autores}

S. S. S.Z.

http://orcid.org/0000-00020708-1216

Universidade Federal da Grande Dourados - Dourados - MS suzanazarske@gmail.com

\section{A. P.}

https://orcid.org/0000-00029051-7550

Universidade Federal da Grande Dourados - Dourados - MS veronica.ufgd.tci@gmail.com

O. M. P.R. R. https://orcid.org/0000-00025332-256X

Universidade Estadual Paulista Júlio de Mesquita Filho - Bauru, SP

olgarolim29@gmail.com.br

\section{Direitos Autorais}

Este é um artigo de acesso aberto e pode ser reproduzido livremente, distribuído, transmitido ou modificado, por qualquer pessoa desde que usado sem fins comerciais. 0 trabalho é disponibilizado sob a licença Creative Commons CC-BY-NC.

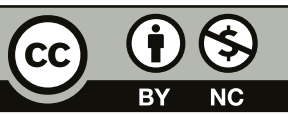




\section{M." INTERACÃO EM PSICOLOGIA}

vos comportamentos, ampliando seu repertório. Aprenderá a realizar os cuidados básicos, discriminar o choro do bebê e identificar suas necessidades buscando respondê-las de forma adequada (Alvarenga et al., 2016). Segundo as autoras, os comportamentos parentais emitidos durante a interação com o bebê se devem a múltiplos aspectos, produtos de variáveis biológicas, da história de vida de cada mãe, das contingências atuais a que está exposta, da sua interação com o ambiente (físico e social) e da sua cultura. Para o bebê, a interação com o cuidador propiciará que ele aprenda novos comportamentos, que podem atuar positiva ou negativamente no seu desenvolvimento social, cognitivo, motor e de linguagem no primeiro ano de vida (Malmberg, 2015).

Para compreender os aspectos dessa relação, a interação entre mãe e bebê torna-se foco importante das pesquisas sobre o desenvolvimento humano. Essa interação pode ser influenciada tanto por características da criança quanto por características maternas (Sameroff, 2009). Considerando as características das crianças, observa-se os fatores de risco biológico como a prematuridade (Chiodelli et al., 2020; Joaquim et al., 2018) e os tipos de temperamento (Chiodelli, 2016; Cassiano \& Linhares, 2015). A mãe também apresenta características que podem influenciar essa interação, como a responsividade, que se refere ao modo como ela modula seu comportamento e, consequentemente, o ambiente físico em resposta às demandas da criança (Alvarenga \& Piccinini, 2007; Alvarenga et al., 2016), e a intrusividade, que não respeita a autonomia da criança (Seixas et al., 2017). Aspectos da saúde emocional materna em seus diversos constructos (Alvarenga et al., 2016; Nardi et al., 2015), como ansiedade (Beltrami et al., 2013), estresse (Alves et al.,2018) e depressão (Greinert et al., 2018; Ramos \& Furtado, 2007, Servilha \& Bussab, 2015) também podem influenciar negativamente na interação mãe-bebê. Além das características da díade, é importante compreender as variáveis contextuais, como a condição socioeconômica que podem influenciar na qualidade da interação. Entre as variáveis socioeconômicas, a literatura tem apontado forte influência relacionada à escolaridade materna e renda familiar, indicando uma correlação positiva entre essas variáveis e melhores condições de desenvolvimento do bebê (Fernandes et al., 2018).

Para fins de análise dos comportamentos maternos e dos bebês durante a interação, uma das possibilidades tem sido o uso de registros videográficos (em situação livre, estruturada ou experimental), com uso de protocolos específicos para sua avaliação (Ainsworth et al., 1978; Alvarenga \& Cerezo, 2013; Crittenden, 1988; Tronick et al., 1978). O uso desses instrumentos possibilita a observação e codificação de comportamentos interativos da mãe e do bebê para posterior análise e avaliação. São instrumentos sistemáticos que contam, inclusive, com uso de pelo menos um juiz para aferir a fidedignidade do observado.
Suzana Saab de Souza Zarske, Veronica Aparecida Pereira e Olga Maria Piazentin Rolim Rodrigues
A observação pode ser feita em situação livre ou estruturada. O paradigma Face-to-face Still-face pode ser observado a partir de uma situação estruturada que tem sido utilizada para analisar a interação de bebês com diferentes adultos significativos, como pai e mães (Braungart-Rieker et al., 2014), mães (Chiodelli et al., 2020; Faria \& Fuertes, 2007; Fuertes et al. 2009, 2011; Seixas et al., 2017) e cuidadoras (Izidoro et al., 2020). A situação de observação é organizada em três episódios, com duração de até três minutos cada, estando adulto e bebê sentados frente a frente (bebê sentado em cadeira de alimentação, de forma confortável e segura). $01^{\circ}$ e o $3^{\circ}$ episódios focalizam a interação adulto-bebê, com orientação para que o adulto interaja com o bebê de forma mais natural possível. No $2^{\circ}$ episódio, que tem início e fim sinalizado pelo experimentador, o adulto permanece com a face impassível, porém voltada para o bebê, que está posicionado à sua frente (Tronick, et al., 1978). Durante o FFSF, não é permitido o uso de brinquedos ou objetos do bebê e nem retirá-lo da cadeirinha (Chiodelli et al., 2020). Um ponto importante desse paradigma é a oportunidade, no Episódio 2, de observar como o bebê se autorregula diante de uma situação de indisponibilidade do parceiro da interação, uma condição de alguma forma estressante.

Como efeito do Still-Face, no Episódio 2, espera-se que os bebês aumentem a emissão de comportamentos de autorregulação e protesto e diminuam os comportamentos de interação positiva (Tronick et al., 1978). Essa alteração pode permanecer por algum tempo após a retomada de interação no $3^{\circ}$ episódio. Outros dois efeitos que podem ser observados referem-se ao carry-over e o recovery effect (efeito de recuperação). No carry-over, o bebê emite respostas mais negativas e menos positivas nas comparações entre os episódios play e reunião. No efeito de recuperação, comparando os comportamentos do bebê no $2^{\circ}$ e $3^{\circ}$ episódios, é possível perceber a retomada da interação (Mesman et al., 2009).

$\mathrm{Na}$ interação com o adulto, a criança passa a desenvolver, de modo progressivo e autônomo, a sua capacidade autorregulatória (Alvarenga \& Piccinini, 2007; Faria \& Fuertes, 2007). Nesse processo, cada membro da díade (mãe-bebê/ou adulto-bebê) desenvolve diferentes formas para regular o comportamento e se adaptar a cada situação vivida, num sistema de aprendizagem e crescimento mútuo (Simões, 2011).

Entende-se como autorregulação os mecanismos usados pelo indivíduo para controlar suas reações emocionais e comportamentais diante de uma estimulação positiva ou negativa (Linhares \& Martins, 2015). Nos processos regulatórios da criança, verifica-se a presença relevante dos pais e cuidadores como correguladores. Pesquisas evidenciam que a mediação social adequada pelo adulto pode promover o desenvolvimento infantil (Gonçalves, 2015; Izidoro et al, 2020; Linhares \& Martins, 2015). Isso indica que tal mediação caracterizada pelas práticas educativas parentais, estratégias utilizadas na 


\section{MLE INTERACÃO EM LTPSICOLOGIA}

Suzana Saab de Souza Zarske, Veronica Aparecida Pereira e Olga Maria Piazentin Rolim Rodrigues

educação e criação dos filhos, podem ser positivas e negativas (Baumrind, 1966). Se positivas, elas são efetivas para as regulações emocionais e comportamentais da criança em desenvolvimento (Guisso et al., 2019).

Importantes para a socialização e controle das crianças, tais práticas têm como papel incentivar a ocorrência de comportamentos infantis adequados e, ainda, evitar os comportamentos considerados inadequados, importantes para o funcionamento da dinâmica familiar e da sociedade em que estes (pais e filhos) se encontram. A análise das práticas utilizadas pelos pais para educar seus filhos possibilita que intervenções sejam propostas auxiliando-os na tarefa de educar seus filhos.

Gomide (2006), desenvolveu um instrumento, o Inventário de Estilos Parentais (IEP) para avaliar sete práticas educativas parentais: duas positivas e cinco negativas, para pais de crianças de cinco anos até a adolescência. Entre as práticas positivas, a Monitoria Positiva destaca-se pela responsividade, definida pela atenção voltada à localização dos filhos, promoção e manutenção da qualidade de suas atividades, bem como à adaptação aos diferentes contextos, envolvendo demonstrações de afeto dos pais. Outra prática positiva é a do Comportamento Moral, que remete ao ensino de valores culturalmente aceitos, como honestidade e senso de justiça, auxiliando a criança na compreensão sobre o que é certo e errado. As práticas negativas são: Negligência, Disciplina Relaxada, Abuso Físico, Monitoria Negativa e Punição Inconsistente. A Negligência caracteriza-se pela ausência de interesse dos pais sobre a educação de seus filhos, sem demonstração de afeto. A Disciplina Relaxada implica no estabelecimento de regras e rotinas, mas cujo cumprimento é sempre relevado. 0 Abuso Físico implica no uso de ameaças, castigos e palmadas para controle do comportamento inadequado infantil. A Punição Inconsistente é influenciada pelo humor dos pais para punir ou reforçar os comportamentos de seus filhos e tem, como consequência, que os filhos aprendem mais a observar seus pais e menos a seguir suas recomendações. A Monitoria Negativa caracteriza-se pelo estabelecimento de regras em excesso e seu não cumprimento resulta em consequências negativas. A somatória das práticas positivas menos a somatória das negativas resulta no índice de estilo parental, apontando que os pais que possuem práticas adequadas, até aqueles que apresentarem mais práticas inadequadas, por serem de risco, necessitam de intervenção imediata para desenvolverem um repertório suficiente de práticas positivas.

Em busca de um instrumento que possibilitasse a caracterização das práticas utilizadas por mães de bebês, Altafim et al. (2008) adaptaram o IEP para aplicação para essa população e desenvolveram o Inventário de Estilos Parentais para Mães de Bebês (IEPMB). A adaptação resultou em um instrumento composto por cinco práticas, uma positiva (Monitoria Positiva) e quatro negativas (negligência, disciplina relaxada, punição inconsistente e abuso físico). Nessa versão adaptada, foram retiradas as dimensões Comportamento Moral e Monitoria Negativa em função da idade das crianças do estudo (bebês até 12 meses), por referirem-se às práticas não observadas nessa fase. Em Monitoria Positiva, prevaleceram questões que avaliam, principalmente, a responsividade materna. Esse instrumento, na forma adaptada, foi utilizado em outros estudos com bebês e mães adolescentes (Altafim et al., 2008; Rodrigues et al., 2011) e adultas (Altafım \& Rodrigues, 2013, 2015; Nogueira et al., 2013), possibilitando a descrição de efeitos de variáveis sociodemográficas sobre as práticas educativas maternas (Rodrigues et al., 2013) e da saúde emocional materna sobre as práticas educativas de mães de bebês (Mangili \& Rodrigues, 2018; Rodrigues \& Nogueira, 2016).

A literatura tem indicado que práticas parentais pautadas na empatia, diálogo, afeto e estabelecimento de limites de forma adequada estão relacionadas ao desenvolvimento de comportamentos como autonomia, respeito e boa convivência em sociedade (Marin et al., 2013). As práticas educativas maternas têm influência nas interações estabelecidas com os bebês desde o nascimento e a análise dessas interações possibilitam o desenvolvimento de práticas mais adequadas e responsivas. Todavia não foram encontrados estudos que analisaram a relação entre as práticas educativas relatadas por mães de bebês e os comportamentos interativos da díade em idades precoces, configurando uma lacuna na literatura. Desse modo, considera-se relevante identificar que a relação entre eles pode dar dicas para subsidiar intervenções que poderiam ocorrer precocemente.

Diante do exposto, a partir dos estudos voltados à compreensão dos comportamentos diádicos na primeira infância e o que os influenciam, o presente estudo teve por objetivos: a) descrever e comparar as práticas parentais de mães de bebês aos três meses; b) descrever as categorias positivas e negativas maternas e, positivas, negativas e de autorregulação do bebê, observadas durante o FFSF; e: b1) investigar o efeito das práticas parentais (positivas e negativas) sobre a interação mãe-bebê; b2) comparar os categorias interativas maternas e dos bebês durante o $1^{\circ}$ e $3^{\circ}$ episódio.

As hipóteses do presente estudo foram estabelecidas a partir dos efeitos do Still-Face (Mesman et al., 2009; Tronick et al. 1978). São esperados os efeitos: Still Face, carry-over e recovery effect. Neste estudo, levantou-se a hipótese de que o efeito carry over seria mais observado no Episódio 3, entre as mães de maior ocorrência de Práticas Negativas, o que indicaria maior dificuldade para retomada da interação. E mães com maior ocorrência de Práticas Parentais Positivas contribuiriam, de forma mais efetiva, para retomada da interação, sem alterações significativas de comportamento de protesto (OSN) e não interativos. 


\section{H MTERAC̄OOEM IT PSICOLOGIA}

\section{MÉTODO}

A pesquisa enquadra-se em um recorte do projeto desenvolvido com o grupo de pesquisa intitulado: Prematuridade: percepção materna, saúde emocional materna, interação mãe-bebê e desenvolvimento infantil, aprovado pelo Comitê de Ética da Universidade Federal da Grande Dourados, parecer $n^{\circ}$ 2.421.209.

\section{PARTICIPANTES}

0 estudo contou com a participação de 39 díades. A seleção dos participantes aconteceu em um Serviço Escola de Psicologia de uma universidade do interior de Mato Grosso do Sul, a partir do contato com familiares que participavam do Projeto "Prematuridade: percepção materna, saúde emocional materna, interação mãe-bebê e desenvolvimento infantil”. Os
Suzana Saab de Souza Zarske. Veronica Aparecida Pereira e Olga Maria Piazentin Rolim Rodrigues

Tabela 1: Caracterização e comparação da amostra de mães e bebês no terceiro mês a partir dos dados sociodemográficos.

\begin{tabular}{|c|c|c|c|c|}
\hline & Frequência & $\%$ & $X^{2}$ & $\mathbf{p}$ \\
\hline Sexo do bebê & & & & \\
\hline Masculino & 20 & 51,3 & \multirow{2}{*}{0,26} & \multirow{2}{*}{0,87} \\
\hline Feminino & 19 & 48,7 & & \\
\hline \multicolumn{5}{|l|}{ Idade Gestacional } \\
\hline Pré-termo & 8 & 20,5 & \multirow{2}{*}{13,56} & \multirow{2}{*}{0,00} \\
\hline A termo & 31 & 79,5 & & \\
\hline \multicolumn{5}{|l|}{ Via de nascimento } \\
\hline Cesárea & 28 & 71,8 & \multirow{2}{*}{7,41} & \multirow{2}{*}{0,01} \\
\hline Parto normal & 11 & 28,2 & & \\
\hline \multicolumn{5}{|l|}{ Idade Materna } \\
\hline Até 29 anos & 21 & 53,8 & \multirow{2}{*}{0,23} & \multirow{2}{*}{0,63} \\
\hline Acima de 30 anos & 18 & 46,2 & & \\
\hline \multicolumn{5}{|l|}{ Escolaridade Materna } \\
\hline Até o Ensino Médio & 14 & 35,9 & \multirow[b]{2}{*}{3,10} & \multirow[b]{2}{*}{0,08} \\
\hline $\begin{array}{l}\text { Ensino Superior e/ou Pós-Gra- } \\
\text { duação }\end{array}$ & 25 & 64,1 & & \\
\hline \multicolumn{5}{|l|}{ Trabalho remunerado } \\
\hline Sim & 31 & 79,5 & 13,56 & \multirow{2}{*}{0,00} \\
\hline Não & 8 & 20,5 & & \\
\hline \multicolumn{5}{|l|}{ Conjugalidade } \\
\hline Com parceiro & 35 & 89,74 & \multirow[t]{2}{*}{35,00} & \multirow[t]{2}{*}{0,00} \\
\hline Sem parceiro & 4 & 10,36 & & \\
\hline \multicolumn{5}{|l|}{ Número de filhos } \\
\hline Primípara & 28 & 71,8 & \multirow{2}{*}{49,29} & \multirow{2}{*}{0,00} \\
\hline Multípara & 11 & 28,2 & & \\
\hline
\end{tabular}

Graus de liberdade: 1,0 para Qui-Quadrado

familiares foram convidados a participar da presente pesquisa e, os que aceitaram, assinaram o Termo de Consentimento Livre e Esclarecido. Na Tabela 1, encontram-se os dados sociodemográficos para a amostra geral. Os bebês apresentaram uma distribuição semelhante para sexo $(p=0,87)$, porém apresentaram diferenças quanto a condição e via de nascimento, com predominância de bebês nascidos a termo $(79,5 \%)$ e via cesárea (71,8\%), com valores de X2 iguais ou inferiores a 0,01. Quanto às variáveis maternas, houve semelhança para idade e escolaridade, considerando os recortes até 29 anos e mais de 30 anos, até o ensino médio ou ensino superior e/ou pós-graduação. As diferenças foram observadas quanto a realização de variáveis trabalho remunerado $(79,5 \%$ das mães exerciam função remunerada), conjugalidade $(89,74 \%$ vivam com o parceiro) e número de filhos (71,8\% eram primíparas). Para essas variáreis, os valores do Qui-Quadrado $\left(X^{2}\right)$ foram inferiores a 0,01. 


\section{M*' INTERACÃO EM L PSICOLOGIA}

Suzana Saab de Souza Zarske, Veronica Aparecida Pereira e Olga Maria Piazentin Rolim Rodrigues

\section{LOCAL}

Para a coleta de dados dos pais e bebês, utilizou-se de uma sala cedida pelo laboratório de uma clínica escola de Psicologia, devidamente equipada com ar-condicionado, cadeiras, mesa, boa iluminação. Nesse laboratório são realizadas atividades de pesquisa e/ou extensão, desenvolvidas pelo corpo técnico e estagiários, estabelecendo parcerias com órgãos governamentais e não governamentais das áreas de saúde, educação, direitos, trabalho e assistência social. Na impossibilidade dos pais em comparecer ao local por razões de transportes e horário de trabalho, a coleta de dados ocorreu em domicílio, na sala da família. Entre as participantes desse estudo, nove delas receberam visita domiciliar $(23,1 \%)$.

\section{MATERIAIS E INSTRUMENTOS}

Para a coleta dos dados sociodemográficos, utilizou-se de uma entrevista semiestruturada, pautada em dados de identificação, histórico gestacional e desenvolvimento atual do bebê.

Para avaliação dos Estilos Parentais, utilizou-se o Inventário de Estilos Parentais para Pais e Mães de Bebês - IEPMPB (Rodrigues et al., 2011) adaptado de Gomide (2006). A partir do IEPMPB, são analisadas cinco práticas parentais, mas não o estilo parental, sendo uma delas voltada à descrição de práticas positivas, à Monitoria Positiva e, quatro práticas Negativas: a) Negligência, b) Disciplina Relaxada, c) Abuso Físico, e d) Punição Inconsistente. Trata-se de um instrumento de auto aplicação, com 25 itens, distribuídos randomicamente. Para essa amostra, foram realizados testes de consistência interna, a partir do Alpha Cronbach, com os seguintes resultados: Geral: 0,612; Monitoria Positiva: 0,543; Negligência: 0,547; Disciplina relaxada: 0,611; Abuso Físico: 0,713; e Punição Inconsistente: 0,456 . Na verificação de consistência interna do Inventário de Estilos Parentais de Gomide (2006), os resultados do Alpha de Cronbach variaram de 0,46 para monitoria negativa materna a 0,866 para comportamento moral paterno (Sampaio \& Gomide, 2017).

Para realização da filmagem, foram utilizadas duas filmadoras com tripé, cadeirinha para bebê e sineta para transição entre os episódios de filmagem. A partir dos resultados das filmagens, utilizou-se o Protocolo do FFSF, de acordo com os padrões apresentados por Seixas et al. (2017). Nesse protocolo, os comportamentos do bebê observados a partir das filmagens foram registrados em episódios de cinco segundos, avaliando comportamentos de Orientação Social Positiva - OSP (olhar, sorrir, vocalizar, alcançar o adulto); Orientação Social Negativa - OSN (resmungar, chorar, arquear o corpo, empurrar o adulto, protestar, apresentar expressão facial negativa, como: franzir a testa, aproximar sobrancelhas e outras expressões que sinalizam o início do choro ou desagrado) e Autorregulação - AUR (desviar o olhar, tocar-se, levar as mãos à boca, sugar as mãos, esfregar mãos ou pés um no outro) (Varão, 2012).

Os comportamentos do adulto foram registrados seguindo os mesmos parâmetros de Orientação Social Positiva - OSP e Orientação Social Negativa - OSN, pautando-se nas categorias descritas por Varão (2012) (OSP - olhar, falar com o bebê, tocar, beijar, fazer cócegas e fazer comentários positivos sobre o bebê; OSN - desviar o olhar, evitar o jogo, fazer comentários negativos sobre o bebê ou sobre o seu comportamento, apresentar expressão facial negativa, como franzir a testa e aproximar sobrancelhas).

\section{PROCEDIMENTO}

Primeiramente, as mães responderam a uma entrevista semiestruturada para obtenção dos dados sociodemográficos, desde o histórico gestacional ao desenvolvimento do bebê. Entre o terceiro e quarto mês de vida do bebê, as mães responderam ao IEPMPB e participaram da filmagem da interação.

O IEPMPB foi aplicado individualmente, antes da filmagem. A pesquisadora permaneceu na sala para esclarecimento de alguma dúvida quanto ao preenchimento, uma vez que o inventário é de autoaplicação.

A filmagem foi realizada em uma sala de espelhos de uma clínica escola ou em espaço individualizado, quando a coleta ocorreu em domicílio. Em ambos os contextos, a experimentadora assegurou que o bebê estivesse alimentado, limpo, sem incômodo relacionado a vacina, dor, febre ou outro desconforto. 0 espaço reservado atendeu às condições de sigilo e ausência de interrupções, buscando eliminar o risco de interferência.

A duração da filmagem foi de até nove minutos, distribuídos em três episódios de até três minutos cada. Bebê e sua mãe foram colocados face a face, estando o bebê em uma cadeirinha confortável que lhe permitiu movimentar pernas e braços livremente. Uma filmadora foi focada no bebê e outra na mãe, permitindo que os vídeos pudessem ser editados posteriormente para exibição simultânea, para análise da interação. No $1^{\circ}$ episódio, a mãe foi orientada a interagir da forma mais natural possível com o bebê. Após um sinal sonoro, emitido pela pesquisadora, a mãe interrompia a interação, mantendo apenas contato visual com o bebê. Incômodos apresentados pelo bebê (choro ou choramingo apresentados durante 15 segundos) levavam à interrupção do episódio antes dos três minutos e iniciava-se o $3^{\circ}$ episódio (caso ocorresse no $1^{\circ}$ episódio, interrompia-se a filmagem). $03^{\circ}$ episódio iniciava com o mesmo som, diante do qual o adulto retomava a interação com o bebê. Não houve necessidade de interrupção da filmagem durante os episódios da presente amostra. 


\section{H. INTERACÃO EM LFICOLOGIA}

Suzana Saab de Souza Zarske, Veronica Aparecida Pereira e Olga Maria Piazentin Rolim Rodrigues

\section{ANÁLISE DE DADOS}

Os dados da entrevista foram organizados por meio de estatística descritiva, apresentados na Tabela 1, com análise de homogeneidade a partir do teste de Levene e do teste do Qui-Quadrado.

Os dados do IEPPMB foram tabulados a partir da identificação das cinco categorias de práticas parentais, quantificando-se a frequência dos comportamentos relatados em escala do tipo likert. As respostas para cada item receberam as pontuações segundo a frequência indicada, de zero a dois, entre nunca, às vezes ou sempre. Dessa forma, cada participante teve um escore geral para o instrumento e escores específicos para as práticas parentais. A tabulação foi realizada a partir dos escores médios obtidos para Práticas Positivas e Práticas Negativas $[(a+b+c+d) / 4]$ (Baixa, Média e Alta). Para Práticas Positivas, foram considerados os seguintes escores arbitrários: Baixa - até 1,60; Média - de 1,61 a 1,80; e Alta - acima de 1,81. Para Práticas Negativas: Baixa - até 0,49; Média - de 0,50 a 1,80; e Alta - acima de 1,81.

Os dados do FFSF foram registrados em protocolos com intervalos de cinco segundos (Chiodelli et al., 2020). Dessa forma, cada episódio poderia conter registros de comportamentos observados em 36 intervalos. As análises de vídeo do FFSF passaram por análise de confiabilidade, por observadores independentes, para $30,77 \%$ da amostra (12 filmagens). Os resultados registrados em protocolo, pelos observadores, foram submetidos à análise de correlação intraclasse, com resultados de 0,870.

Realizou-se análise multivariada, considerando os efeitos das Práticas Positivas e Negativas (isoladas ou em interação) como variáveis preditoras e as médias observadas para as categorias do FFSF como variáveis dependentes. Foram geradas as estatísticas descritivas para as categorias do FFSF e as mesmas foram submetidas aos efeitos das práticas parentais, devidamente classificadas em Positiva ou Negativa (Alta, Média ou Baixa). A partir das estatísticas descritivas das categorias do FFSF, foram comparados os resultados observados para $1^{\circ}$ e $3^{\circ}$ episódio, dos comportamentos maternos e dos bebês, e, para avaliação do efeito de retomada da interação, foram comparados os episódios 2 e 3 . Para essas comparações, foi utilizado o teste de Wilcoxon.

As variáveis dependentes (categorias do FFSF) não apresentaram uma distribuição normal, por isso, foram observadas as condições de homogeneidade pelo teste de Levene (Pestana \& Gageiro, 2014), com a inserção das variáveis independentes como fatores (Práticas Positivas e Práticas Negativas). Houve homogeneidade de variância para as categorias dos comportamentos dos bebês, considerando o Fator Práticas Positivas. (OSP $1^{\circ}$ episódio: $F=0,939 ; p=0,401 ; 2^{\circ}$ episódio: $F=0,593 ; p=0,558,3^{\circ}$ episódio: $F=1,529 ; p=0,231 ;$ OSN $1^{\circ}$ episódio: $F=2,442 ; p=0,102,2^{\circ}$ episódio $F=1,499 ; p=0,237$, $3^{\circ}$ episódio $F=0,001 ; p=0,999$, AUR $1^{\circ}$ episódio $F=0,624 ; p=$ $0,542,2^{\circ}$ episódio $F=0,726 ; p=0,491$, e $3^{\circ}$ episódio $F=2,119$; $p=0,135)$. Também foi observada homogeneidade de variância para as categorias dos comportamentos maternos (OSP $1^{\circ}$ episódio $F=0,864 ; p=0,430,3^{\circ}$ episódio $F=2,094 ; p=0,138$; e OSN $1^{\circ}$ episodio $F=0,684 ; p=0,511,3^{\circ}$ episódio $(F=1,717 ; p$ $=0,194)$. 0 critério da homocedasticidade não foi aplicado por se tratar de grupos com número de participantes semelhante entre os fatores.

\section{RESULTADOS}

Os resultados obtidos para as 25 questões do IEPMPB foram primeiramente organizados em duas categorias, Prática Positiva e Prática Negativa, conforme observa-se na Tabela 2. A comparação das médias, a partir do teste $t$ de Student, indicou diferença significativa, com médias superiores para Prática Positiva. Foram feitas subdivisões das mães em grupos, com base na pontuação média das participantes, Alta, Média e Baixa, em cada uma das categorias, Prática Positiva e Prática Negativa. Observa-se que os grupos apresentaram composições semelhantes entre o número de participantes. Em relação às subcategorias da Prática Negativa, observou-se média superior para Disciplina Relaxada $(0,83)$. 
Tabela 2: Análise descritiva das práticas parentais a partir do IEPMPB.

\begin{tabular}{llll}
\hline Categorias & Média (DP) & Mínimo-Máximo & $p$ \\
\hline Prática Positiva & $1,76(0,27)$ & $1,0-2,00$ & 0,000 \\
\hline Prática Negativa & $0,87(0,56)$ & $0,0-1,80$ & \\
\hline & Prática Positiva & Prática Negativa & \\
& $\mathbf{n}(\%)$ & $\mathbf{n}(\%)$ & \\
Baixa & $11(28,2)$ & $13(33,3)$ & \\
Média & $12(30,8)$ & $12(30,8)$ & \\
Alta & $16(41,0)$ & $14(35,9)$ & \\
\hline Subcategorias da Prática Negativa & Média (DP) & Mínimo-Máximo & \\
\hline Negligência & $0,27(0,32)$ & $0,0-1,25$ & \\
Disciplina Relaxada & $0,83(0,46)$ & $0,0-2,00$ & \\
Abuso Físico & $0,04(0,09)$ & $0,0-0,40$ & \\
Punição Inconsistente & $0,22(0,21)$ & $0,0-0,67$ & \\
\hline
\end{tabular}

Notas: $p=0,000$ - significativo para o intervalo de confiança de $95 \%$ observado a partir do teste $t$.

Os resultados das categorias de interação observadas durante o FFSF foram organizados a partir da pontuação média (Alta, Média e Baixa) para cada um dos tipos de Prática Positiva ou Negativa. Os dados encontram-se apresentados nas Tabelas 3 e 4.

$\mathrm{Na}$ Tabela 3, encontram-se os dados descritivos e comparativos do FFSF, para a categoria Prática Positiva, dos comportamentos dos bebês (Orientação Social Positiva - OSPbb, Orientação Social Negativa - OSNbb e Autorregulação - AUR) e das mães (OSPmãe e OSNmãe), considerando a média e o desvio padrão observados por episódio, para os três grupos analisados (Alta, Média ou Baixa). A Anova de uma via, utilizada para comparar os grupos, mostrou que houve efeito da Prática Positiva sobre a Autorregulação do bebê no $2^{\circ}$ episó- dio $[F(2,0,6,71)=3,426 ; p<0,05]$, com médias superiores para Prática Positiva Alta. 0 teste de Post hoc de Sidak indicou diferença entre as médias dos grupos Alta e Baixa $(p<0,05)$. Em relação à comparação entre os dados observados entre $01^{\circ}$ e $3^{\circ}$ episódio do FFSF, observou-se um aumento significativo de OSNbb para os grupos Baixa e Média ( $p=0,022$ e 0,023, respectivamente). Para o grupo Alta, houve diminuição significativa de OSPbb $(p=0,035)$. Para as categorias maternas, não houve diferença significativa na comparação entre $1^{\circ}$ e $3^{\circ}$ episódio. Quanto aos comportamentos de AUR, os bebês dos grupos cujas mães tem frequência Média e Alta diminuíram significativamente do $2^{\circ}$ para o $3^{\circ}$ episódio. Para o grupo de frequência Baixa não houve diferença entre o $2^{\circ}$ e $\circ 3^{\circ}$ episódio, apresentando frequência semelhante de AUR nos dois episódios. 
Tabela 3: Descrição das categorias do FFSF e análise do efeito das Práticas Parentais Positivas.

\begin{tabular}{|c|c|c|c|c|c|}
\hline \multirow{3}{*}{ Categorias FFSF } & \multicolumn{5}{|c|}{$\begin{array}{l}\text { Práticas Parentais } \\
\text { Positivas }\end{array}$} \\
\hline & Baixa (n11) & Média (n12) & Alta (n16) & $\Gamma$ & 0 \\
\hline & $M(D P)$ & $M(D P)$ & $M(D P)$ & $r$ & $r$ \\
\hline OSPbb $1^{\circ}$ episódio & $1,56(0,67)$ & $1,41(0,75)$ & $1,63(0,59)$ & 0,402 & 0,672 \\
\hline OSPbb $2^{\circ}$ episódio & $0,90(0,54)$ & $1,41(0,75)$ & $0,89(0,46)$ & 0,003 & 0,997 \\
\hline OSPbb $3^{\circ}$ episódio & $1,25(0,42)$ & $1,26(0,65)$ & $1,11(0,79)$ & 0,215 & 0,808 \\
\hline $1^{\circ} \times 3^{\circ}$ episódios $-p$ & 0,060 & 0,069 & 0,035 & & \\
\hline $2^{\circ} \times 3^{\circ}$ episódios $-p$ & 0,110 & 0,064 & 0,334 & & \\
\hline OSNbb $1^{\circ}$ episódio & $0,07(0,13)$ & $0,18(0,22)$ & $0,19(, 027)$ & 1,004 & 0,377 \\
\hline OSNbb $2^{\circ}$ episódio & $0,41(0,61)$ & $0,39(0,46)$ & $0,57(0,63)$ & 0,407 & 0,669 \\
\hline OSNbb $3^{\circ}$ episódio & $0,47(0,63)$ & $0,58(0,75)$ & $0,49(0,58)$ & 0,085 & 0,919 \\
\hline $1^{\circ} \times 3^{\circ}$ episódios $-p$ & 0,022 & 0,023 & 0,066 & & \\
\hline $2^{\circ} \times 3^{\circ}$ episódios $-p$ & 0,859 & 0,389 & 0,814 & & \\
\hline AUR $1^{\circ}$ episódio & $0,56(0,43)$ & $0,69(0,37)$ & $0,73(0,40)$ & 0,572 & 0,569 \\
\hline AUR $2^{\circ}$ episódio & $0,93(0,50)$ & $0,90(0,38)$ & $1,33(0,43)$ & 3,426 & 0,046 \\
\hline AUR $3^{\circ}$ episódio & $0,61(0,21)$ & $0,70(0,32)$ & $0,79(0,48)$ & 0,699 & 0,504 \\
\hline $1^{\circ} \times 3^{\circ}$ episódios $-p$ & 0,477 & 0,249 & 0,572 & & \\
\hline $2^{\circ} \times 3^{\circ}$ episódios - $p$ & 0,075 & 0,039 & 0,009 & & \\
\hline OSPmãe $1^{\circ}$ episódio & $2,46(0,56)$ & $2,53(0,62)$ & $2,95(0,54)$ & 2,917 & 0,067 \\
\hline OSPmãe $3^{\circ}$ episódio & $2,33(0,54)$ & $2,38(0,71)$ & $2,39(0,95)$ & 0,020 & 0,980 \\
\hline $1^{\circ} \times 3^{\circ}$ episódios $-p$ & 0,433 & 0,753 & 0,055 & & \\
\hline OSNmãe $1^{\circ}$ episódio & $0,05(0,10)$ & $0,11(0,12)$ & $0,07(0,11)$ & 0,961 & 0,392 \\
\hline OSNmãe $3^{\circ}$ episódio & $0,09(0,09)$ & $0,07(0,08)$ & $0,12(0,26)$ & 0,214 & 0,808 \\
\hline $1^{\circ} \times 3^{\circ}$ episódios $-p$ & 0,091 & 0,248 & 0,779 & & \\
\hline
\end{tabular}

Notas: Graus de liberdade: 2,0. $p<0,05 . M=$ Média; $D P=$ Desvio Padrão. * Post hoc de Sidac - Prática Positiva Alta > Baixa e Média. Graus de liberdade: 2,0. $p<0,05 . M=$ Média; $D P=$ Desvio Padrão. Análises intergrupos - Anova; análises intragrupos $\left(1^{\circ}\right.$ e $3^{\circ}$ episódio $)-W i l c o x o n$.

Do mesmo modo, foram geradas as médias das categorias do FFSF considerando as divisões entre as díades a partir dos escores médios para Práticas Negativas (Alta, Média e Baixa), conforme observa-se na Tabela 4. Os testes realizados a partir da Anova não indicaram efeitos das práticas parentais negativas sobre a interação. As comparações entre o $1^{\circ}$ e o $3^{\circ}$ episódio do FFSF, a partir do Wilcoxon, mostraram algumas diferenças para as categorias de comportamentos dos bebês. Para OSPbb, houve diminuição significativa para os grupos Média e Alta ( $p=0,009$ e 0,028 , respectivamente). Para OSNbb, houve aumento significativo para os três grupos $(p=0,047$ Baixa, 0,038 Média e 0,015 Alta). Para os comportamentos de AUR, os bebês dos grupos de mães com práticas parentais negativas Baixa ou Alta, houve redução significativa do $2^{\circ}$ para o $3^{\circ}$ episódio. Para o grupo de mães com prática parental negativa Média, não houve diferença entre o $2^{\circ}$ e o $3^{\circ}$ episódios, apresentando frequência semelhante de AUR nos dois episódios. Não houve diferença significativa para as categorias de comportamentos maternos (OSPmãe e OSNmãe). 
Tabela 4: Descrição das categorias do FFSF e análise do efeito das Práticas Parentais Negativas.

\begin{tabular}{|c|c|c|c|c|c|}
\hline \multirow{3}{*}{ Categorias FFSF } & \multicolumn{5}{|c|}{ Práticas Parentais Negativas } \\
\hline & Baixa (n14) & Média (n12) & Alta (n13) & $\Gamma$ & $\Omega$ \\
\hline & $M(D P)$ & $M(D P)$ & $(\mathrm{M}(\mathrm{DP})$ & $r$ & $P$ \\
\hline OSPbb $1^{\circ}$ episódio & $1,67(0,44)$ & $1,67(0,79)$ & $1,30(0,68)$ & 1,362 & 0,269 \\
\hline OSPbb $2^{\circ}$ episódio & $1,04(0,56)$ & $0,90(050)$ & $0,74(0,42)$ & 1,133 & 0,333 \\
\hline OSPbb $3^{\circ}$ episódio & $1,24(0,61)$ & $1,08(0,58)$ & $1,26(0,76)$ & 0,249 & 0,781 \\
\hline $1^{\circ} \times 3^{\circ}$ episódios $-p$ & 0,507 & 0,009 & 0,028 & & \\
\hline $2^{\circ} \times 3^{\circ}$ episódios $-p$ & 0,055 & 0,208 & 0,311 & & \\
\hline OSNbb $1^{\circ}$ episódio & $0,14(0,19)$ & $0,15(0,25)$ & $0,17(0,23)$ & 0,095 & 0,909 \\
\hline OSNbb $2^{\circ}$ episódio & $0,44(0,57)$ & $0,36(0,52)$ & $0,58(0,62)$ & 0,574 & 0,568 \\
\hline OSNbb $3^{\circ}$ episódio & $0,39(0,38)$ & $0,47(0,67)$ & $0,67(0,82)$ & 0,659 & 0,524 \\
\hline $1^{\circ} \times 3^{\circ}$ episódios $-p$ & 0,047 & 0,038 & 0,015 & & \\
\hline $2^{\circ} \times 3^{\circ}$ episódios $-p$ & 0,638 & 0,594 & 0,807 & & \\
\hline AUR $1^{\circ}$ episódio & $0,56(0,41)$ & $0,68(0,48)$ & $0,76(0,31)$ & 0,765 & 0,473 \\
\hline AUR $2^{\circ}$ episódio & $0,98(0,56)$ & $1,16(0,44)$ & $1,10(0,43)$ & 0,456 & 0,638 \\
\hline AUR $3^{\circ}$ episódio & $0,61(0,28)$ & $0,85(0,50)$ & $0,69(0,28)$ & 1,365 & 0,269 \\
\hline $1^{\circ} \times 3^{\circ}$ episódios $-p$ & 0,433 & 0,750 & 0,422 & & \\
\hline $2^{\circ} \times 3^{\circ}$ episódios $-p$ & 0,013 & 0,075 & 0,028 & & \\
\hline OSPmãe $1^{\circ}$ episódio & $2,84(0,48)$ & $2,60(0,64)$ & $2,58(0,68)$ & 0,735 & 0,487 \\
\hline OSPmãe $3^{\circ}$ episódio & $2,53(0,66)$ & $2,09(0,88)$ & $2,47(0,72)$ & 1,254 & 0,298 \\
\hline $1^{\circ} \times 3^{\circ}$ episódios $-p$ & 0,917 & 0,075 & 0,152 & & \\
\hline OSNmãe $1^{\circ}$ episódio & $0,07(0,11)$ & $0,10(0,13)$ & $0,07(0,09)$ & 0,297 & 0,745 \\
\hline OSNmãe $3^{\circ}$ episódio & $0,16(0,26)$ & $0,09(0,12)$ & $0,03(0,49)$ & 1,828 & 0,176 \\
\hline $1^{\circ} \times 3^{\circ}$ episódios $-p$ & 0,262 & 0,423 & 0,248 & & \\
\hline
\end{tabular}

Fonte: Elaborada pelas autoras.

Graus de liberdade: $2,0 .{ }^{*} p<0,05$.

A análise multivariada da influência das Práticas Positivas e Negativas sobre as categorias de interação do FFSF, apresentada na Tabela 5, indicou efeitos no $3^{\circ}$ episódio, para categoria OSP tanto dos bebês como de suas mães, indicando desempenhos superiores para os grupos formados por mães com relatos de Práticas Positivas Médias e Práticas Negativas Baixas, sendo: $[F(4,10,20)=3,415 ; p<0,05]$ para OSPbb e $[F(4$, $0,415)=4,535 ; p<0,001]$ para OSPmãe. 
Tabela 5: Efeito das Práticas Positivas e Negativas sobre as categorias de interação observadas durante o FFSF.

\begin{tabular}{lllll}
\hline Variável Dependente & Gl & $F$ & $P$ & Post hoc \\
\hline OSPbb episódio 1 & 4 & 1,088 & 0,380 & - \\
OSPbb episódio 2 & 4 & 0,259 & 0,902 & - \\
OSPbb episódio 3 & 4 & 3,415 & $0,021^{*}$ & $\begin{array}{l}\text { > Prática + média e negativa } \\
\text { baixa. }\end{array}$ \\
OSPmãe episódio 1 & 4 & 1,367 &, 269 & \\
OSPmãe episódio 3 & 4 & 4,535 & $0,006^{* *}$ & $\begin{array}{l}\text { > Prática + média e negativa } \\
\text { baixa. }\end{array}$ \\
\hline
\end{tabular}

Fonte: Elaborada pelas autoras.

Graus de liberdade (GL): 4,0. * $p<0,05$, ** $p<0,01$

\section{DISCUSSÃO}

A análise das práticas educativas dessa amostra mostrou que as mães relataram utilizar significativamente mais práticas positivas do que negativas com seus bebês. Tais resultados confirmaram os encontrados por Altafim et al. (2008), Rodrigues et al. (2011), Nogueira et al. (2013), Altafim e Rodrigues (2015), Rodrigues e Nogueira (2016) e Mangili e Rodrigues (2018). Considerando os apontamentos de Guisso et al. (2019) sobre o efeito positivo dessas práticas sobre a regulação emocional, buscou-se analisar os comportamentos interativos da díade, considerando separadamente três grupos com Alta, Média e Baixa frequência de comportamentos tanto positivos como negativos.

As Práticas Positivas envolvem um conjunto de respostas importantes para o cuidado, como responsividade e sensibilidade materna para atendimento das necessidades do bebê (Alvarenga \& Piccinini, 2007). Das práticas negativas, a mais frequente foi a Disciplina Relaxada, um resultado encontrado por Mangili e Rodrigues (2018), Rodrigues e Nogueira (2016) e Altafim e Rodrigues (2015). Ela é caracterizada pelo não cumprimento de regras e padrões inconsistentes no comportamento da mãe, presente nas práticas maternas da amostra, e pode ser exemplificada a partir de comportamentos como: pegar ou não o bebê no colo, atendê-lo prontamente em livre demanda para as mamadas e em outros momentos demorar muito para atender, sem que o bebê consiga prever os comportamentos maternos contingentes ao seu choro. É importante considerar a tenra idade dos bebês (três meses) e o tempo de adaptação da mãe (tanto fisicamente como o cansaço de noites mal dormidas e constantes demandas do bebê) que a fazem ceder ao choro.

$\mathrm{Na}$ comparação das categorias de interação observadas durante o FFSF, a partir das práticas parentais positivas, observou que os bebês com mães dos grupos Baixa e Média Prática Positiva apresentaram significativamente mais com- portamentos de OSN na retomada da interação ( $3^{\circ}$ episódio), porém não aumentaram a ocorrência de autorregulação. Em contrapartida, os bebês de mães do grupo Alta Prática Positiva não apresentaram diferenças significativas para OSN e AUR, embora tenham diminuído OSP. A análise de variância indicou ainda que esse grupo apresentou significativamente mais comportamentos autorregulatórios durante o $2^{\circ}$ episódio, e que possivelmente contribuiu para melhor retomada. Os achados confirmaram parcialmente as hipóteses iniciais, pautadas nos efeitos descritos por Tronick et al. (1978) e Mesman et al. (2009). As mães mantiveram os comportamentos de OSP durante a retomada de interação, e, para o grupo com práticas parentais mais positivas, houve melhores condições de autorregulação no episódio não interativo, confirmando as indicações de Guisso et al. (2009).

Já em relação à subdivisão por práticas parentais negativas, observou-se um efeito carry-over (Mesman et al. 2009) para os bebês dos grupos Média e Alta Prática Negativa, com aumento significativo dos comportamentos de OSN e diminuição significativa de OSP. Já o grupo de Baixa Prática Negativa teve dados semelhantes aos de Baixa e Média Prática Positiva (aumentaram OSN, mas não AUR). A análise de variância não indicou efeitos da Prática Negativa sobre a interação, possivelmente pelo fato de as mães apresentarem baixas frequências para essas práticas. 0 fato de as mães apresentarem mais práticas positivas que negativas possivelmente encontra-se relacionado ao fato de a mãe tentar garantir, com seus bebês, a boa retomada da interação no $3^{\circ}$ episódio. As mães retomaram a interação com comportamentos semelhantes ao início da interação e, ainda que a maioria dos bebês apresentasse aumento significativo de OSN, isso não fez com que aumentasse também a emissão de respostas negativas, o que demostrou acolhimento e responsividade diante da condição do bebê.

Considerando o efeito da interação entre a coexistência de práticas parentais positivas e negativas, observou-se que elas 


\section{M." INTERACÃO EM PSICOLOGIA}

exerceram influência sobre os comportamentos de Orientação Social Positiva, tanto dos bebês como das mães, no $3^{\circ}$ episódio (de retomada da interação). 0 dado chama atenção para o fato de que nessa relação não são as mães com maiores pontuações para Prática Parental Positiva e, sim, aquelas que, apesar de ficarem na classificação mediana, têm índices baixos de Prática Parental Negativa. Bornstein e Manian (2013) e Moore et al. (2016) analisando comportamentos maternos na interação com seus bebês que, mães altamente positivas, pode sugerir rigidez na interação. Nesse sentido, Moore et al. (2016) ponderaram que níveis moderados na interação mãe-criança podem ser preferíveis a níveis muito elevados. Bornstein e Manian (2013) também fizeram recomendações nessa direção, mas considerando a contingência entre o comportamento materno e da criança. Eles destacaram que níveis moderados de respostas maternas contingentes aos comportamentos dos bebês são preferíveis a níveis muito elevados ou não contingentes.

As práticas positivas são apontadas como uma estratégia frequentemente utilizada pelas mães nos primeiros meses de vida. Por estarem fortemente relacionadas aos cuidados com o bebê, essas práticas caracterizam-se pela preocupação com a localização dos filhos e afetividade, sendo assim, importantes para o estabelecimento de um bom vínculo entre díade (Alvarenga et al., 2016).

Considerando a idade dos bebês, é importante atentar-se aos resultados verificados por Rodrigues et al. (2011) no período de 1 a 4, de 5 a 8 e de 9 a 12 meses de idade, indicando que as Práticas Negativas, como disciplina relaxada e negligência, eram mais frequentes com bebês de mais idade, sugerindo que, à medida em que o bebê cresce, as mães passam a utilizar mais práticas negativas. Considerando que a maioria das mães do presente estudo são trabalhadoras e no período da pesquisa encontravam-se justamente diante do desafio da retomada da atividade laboral, período em que as mães vivenciam uma certa carga de estresse, os resultados deste estudo podem ser tomados como um alerta, no sentido de orientar práticas que possam ser fortalecidas.

Outro fator a ser considerado é a fase inicial da construção da relação entre mãe e filho. A mãe encontra-se diante da tarefa de compreender as preferências do filho, aprendendo a discriminar os seus sinais e a estabelecer sua rotina, que faz com que seus recursos e estratégias em contê-lo (no caso pós-efeito Still Face) sejam limitadas. Altafim e Rodrigues (2015) apontaram que esse período de relações precoces seria um ótimo momento para a realização de intervenções que visem aprimorar o relacionamento mãe-criança, e consequentemente atuarem na prevenção e promoção da saúde e do desenvolvimento.

Baseando-se a perspectiva do modelo transacional proposto por Sameroff (2009), é possível considerar que as relações parentais relatadas pelas mães do presente estudo tiveram
Suzana Saab de Souza Zarske, Veronica Aparecida Pereira e Olga Maria Piazentin Rolim Rodrigues

efeitos positivos, tanto na forma de interagir livremente com os filhos ( $1^{\circ}$ episódio do FFSF), no modo como reagiram ao episódio não interativo ( $2^{\circ}$ episódio) e no episódio de reunião ( $3^{\circ}$ episódio), no qual os bebês retomam a interação com suas mães.

Pesquisas futuras poderão investigar a influência das variáveis contextuais, no âmbito das práticas educativas e no processo de vinculação e possibilitar a comparação de diferentes grupos e características populacionais. Poderão, ainda, ampliar a idade dos bebês a serem investigados, seja para intervir preventivamente em relação às práticas parentais, seja para favorecer interações positivas que contribuam para uma base segura de apoio à criança, com boa qualidade de vinculação. 0 estudo contou com a presença de variáveis heterogêneas na composição amostral (maioria das mães com trabalho remunerado, presença do parceiro, primíparas e que tiveram seus filhos via cesariana) que não foram investigadas no presente estudo. Estudos com populações maiores poderão verificar também a influência dessas variáveis em relação às práticas educativas e suas relações com a interação mãe-bebê.

\section{FINANCIAMENTO}

A pesquisa relatada no manuscrito foi financiada pela bolsa CAPES, de mestrado, concedida à primeira autora.

\section{DECLARAÇÃO DA CONTRIBUIÇÃO DOS AUTORES}

Certificamos que todos os autores participaram suficientemente do trabalho para tornar pública sua responsabilidade pelo conteúdo. A contribuição de cada autor pode ser atribuída como se segue:

S.S.S.Z., V.A.P. e O.M.P.R.R. contribuíram para a conceitualização; S.S.S.Z. e V.A.P. coleta e análise de dados, investigação e obtenção de financiamento; S.S.S.Z fez a redação inicial do artigo; S.S.S.Z., V.A.P., e O.M.P.R.R. são responsáveis pela redação final (revisão e edição).

\section{AGRADECIMENTOS}

As autoras agradecem aos estagiários do Núcleo Comum de Psicologia da UFGD, do ano de 2019, pelo apoio na coleta de dados.

\section{DECLARAÇÃO DE CONFLITOS DE INTERESSE}

Os autores declaram que não há conflitos de interesse no manuscrito submetido. 


\section{H MTERAC̄OOEM PSICOLOGIA}

\section{REFERÊNCIAS}

Ainsworth, M. D. S., Blehar, M. C., Waters, E., \& Wall, S. N. (1978). Patterns of attachment: A psychological study of the Strange Situation. Lawrence Erlbaum.

Altafim, E. R. P., \& Rodrigues, O.M.P.R. (2013). Relacionamento mãe-bebê: estratégias utilizadas na educação e cuidado dos filhos. Movimenta, 6(3), 503-504.

Altafim, E. R. P., \& Rodrigues, O.M.P.R. (2015) Maternal educational practices during the first year of life. Journal of Human Growth and Development, 25(3), 257-262. http:// dx.doi.org/10.7322/jhgd.105999 https://doi.org/10.7322/ jhgd.105999

Altafim, E. R. P., Schiavo, R. A., \& Rodrigues, O. M. P. R. (2008). Práticas parentais de mães adolescentes: um estudo exploratório. Temas sobre Desenvolvimento, 16(93), 104-110.

Alvarenga, P. A., Weber, I. n. d., \& Bolsoni-Silva, A. T. (2016) Cuidados parentais e desenvolvimento socioemocional na infância e na adolescência: uma perspectiva analítico-comportamental. Revista Brasileira de Terapia Comportamental e Cognitiva, 18(1), 4-21. https://doi.org/10.31505/rbtcc. v18i1.827

Alvarenga, P., \& Piccinini, C. A. (2007). O impacto do temperamento infantil, da responsividade e das práticas educativas maternas nos problemas de externalização e na competência social da criança. Psicologia: Reflexão e Crítica, 20(2), 314-323. https://doi.org/10.1590/S010279722007000200018

Alvarenga, P., \& Cerezo, M. Á. (2013). Interação mãe-criança: fidedignidade da versão brasileira do sistema observacional CITMI-R. Avaliação Psicológica, 12(3), 307-316.

Alves, G. M. A. N., Rodrigues, O. M. P. R., \& Cardoso, H. F. (2018). Indicadores emocionais de mães de bebês com risco para o desenvolvimento. Pensando familias, 22(2), 70-87.

Arpini, D. M., Zanatta, E., Marchesan, R. Q., Faraj, S. P., Ledur, C. S., \& Mozzaquatro, C. O. (2016). Interação mãe-bebê: um processo de descobertas. Interação em Psicologia, 19(1), 1-11. https://doi.org/10.5380/psi.v19i1.32503

Baumrind, D. (1966). Effects of authoritative control on child behavior. Child Development, 37(4), 887-907. https://doi. org/10.2307/1126611

Beltrami, L., Moraes, A., \& Souza, A. (2013). Ansiedade materna puerperal e risco para o desenvolvimento infantil. Distúrbios da Comunicação, 25(2), 229-239.

Bornstein, M.H., \& Manian, N. (2013). Responsiveness and Sensitivity Re-Considered: Some Is More. Development and Psychopathology, 25(4pt1), 957-971. https://doi.org/10.1017/ S0954579413000308
Braungart-Rieker, J. M., Zenall, S., Lickenbrock, D. M., Ekas, N.V., Oshio, T., \& Planalp, E. (2014). Attachment in the Making: Mother and Father Sensitivity and Infants' Responses during the Still-Face Paradigm. Journal of Experimental Child Psychology, 125, 63-84. https://doi.org/10.1016/j. jecp.2014.02.007

Cassiano, R. G. M., \& Linhares, M. B. M. (2015). Temperamento, prematuridade e comportamento interativo mãe-criança. Psicologia: Reflexão e Critica, 28(2), 416-424. https://doi. org/10.1590/1678-7153.201528222

Chiodelli, T. (2016). Temperamento e prematuridade: influências sobre a interação mãe bebê. [Dissertaçãode Mestrado, Universidade Estadual Paulista Julio de Mesquita Filho] Repositório Científico da Universidade Estadual Paulista. https://repositorio.unesp.br/handle/11449/144449

Chiodelli, T., Rodrigues, O. M. P. R., Pereira, V. A., Santos, P. L. dos, \& Fuertes, M. (2020). Interactive behaviors between mothers and their prematurely born infants in the face-to-face Still-Face Paradigm. Estudos de Psicologia (Campinas), 37(e180164), 1-13. https://doi.org/10.1590/ 1982-0275202037e180164

Crittenden, P.M. (1988). Relationships at risk. In J. Belsky \& T. Nezworski (Orgs.), The clinical implications of attachment (pp.136-174). Lawrence Erlbaum.

Faria, A., \& Fuertes, M. (2007). Reactividade infantil e a qualidade da interacção mãe-filho. Análise Psicológica, 25(4), 613-623. https://doi.org/10.14417/ap.470

Fernandes, R. C., Manera, F., Boing, L., \& Höfelmann, D. A. (2018). Desigualdades socioeconômicas, demográficas e obstétricas na insegurança alimentar em gestantes. Revista Brasileira de Saúde Materno Infantil, 18(4), 825-834. https://doi.org/10.1590/1806-93042018000400008_

Fuertes, M., Beeghly, M., Lopes-dos-Santos, P., \& Tronick, E. (2011). Predictors of infant positive, negative and self-direct coping during face to face still-face in a Portuguese preterm sample. Análise Psicológica, 29(4), 553-565. https://doi.org/10.14417/ap.103

Fuertes, M., Lopes-dos-Santos, P., Beeghly, M. \& Tronick, E. (2009). Infant coping and maternal interactive behavior predict attachment in a Portuguese sample of healthy preterm infants. European Psychologist, 14(4), 320-331. https://doi. org/10.1027/1016-9040.14.4.320

Gomide, P. I. C. (2006). Inventário de estilos parentais: modelo teórico, manual de aplicação e interpretação. Vozes.

Gonçalves, I. M. G. (2015). Estudo sobre a qualidade da autorregulação do bebé na situação face-to-face-still-face com o pai. [Dissertação de Mestrado, Instituto Politécnico de Lisboa ]. Repositório Científico do Instituto Politécnico de Lisboa. http://hdl.handle.net/10400.21/5619

Guisso, L., Bolze, S. D. A., \& Viera, M. L. (2019). Práticas parentais positivas e programas de treinamento parental: uma revisão sistemática da literatura. Contextos Clínicos, 12(1), 225-25. https://doi.org/10.4013/ctc.2019.121.10 
Greinert, B. R. M., Carvalho, E. R., Capel, H., Marques, A. G., \& Milani, R. G. (2018). A relação mãe-bebê no contexto da depressão pós-parto: estudo qualitativo. Saúde e Pesquisa, 11(1), 81-88. http://dx.doi.org/10.177651/1983-1870

Izidoro, I. R., Pereira, V. A., \& Rodrigues, O. M. P. R. (2020). Transição para educação infantil: estudo comparativo do processo de vinculação primária e secundária. Psico, 51(2), e34869. https://doi.org/10.15448/1980-8623.2020.2.34869

Joaquim, R. H. V. T., Wernet, M., Leite, A. M., Fonseca, L. M. M., \& Mello, D. F. (2018). Interações entre mães e bebês prematuros: enfoque nas necessidades essenciais. Cadernos Brasileiros de Terapia Ocupacional, 26(3), 580-589. https://doi. org/10.4322/2526-8910.ctoA01051

Linhares, M. B. M., \& Martins, C. B. S. (2015). O processo da autorregulação no desenvolvimento de crianças. Estudos de Psicologia (Campinas), 32(2), 281-293. https://doi.org/10.1590/ 0103-166x2015000200012

Malmberg, L. E.; Lewis, S.; West, A.; Murray, E.; Kylva, S. K.; \& Stein, $A$. The influence of mothers' and fathers' sensitivity in the first year of live on children'a cognitive outcomes at 18 and 36 months. Child: care, health and development, 42(1), 1-7, 2015. https://doi.org/10.11111/cch.12294

Mangili, V. R., \& Rodrigues, O.M.P.R. (2018) A influência da depressão pós-parto sobre as práticas educativas parentais. Contextos Clínicos, 11(3), 310-318. http://dx.doi.org/10.4013/ ctc. 2018.113 .03

Marin, A. H., Martins, G. D. F., Freitas, A. P. C. De O., Silva, I. M., Lopes, R. de C. S., \& Piccinini, C. A. (2013). Transmissão Intergeracional de Práticas Educativas Parentais: evidências empíricas. Psicologia: Teoria e Pesquisa, 29(2), 123-132. https:// doi.org/10.1590/S0102-37722013000200001

Mesman, J., van IJzendoorn, M. H., \& Bakermans-Kranenburg, M. J. (2009). The many faces of the Still-Face Paradigm: A review and meta-analysis. Developmental Review, 29(2), 120-162.

Moore, G. A., Quigley, K. M., Voegtline, K. M., \& DiPietro, J. A. (2016). Don't worry, be (moderately) happy: Mothers' anxiety and positivity during pregnancy independently predict lower mother-infant synchrony. Infant Behavior and Development, 42, 60-68. https://doi.org/10.1016/j.infbeh.2015.11.002

Nardi, C. G. A., Rodrigues, O. M. P. R., Melchiori, L. E., Salgado, M. H., Tavano, L. D. A. (2015). Bebês com Sequência de Pierre Robin: saúde mental materna e interação mãe-bebê. Estudos de Psicologia (Campinas), 32(1), 129-140. https://doi.org/10.1590/0103-166X2015000100012.

Nogueira, S. C., Altafim, E. R. P., \& Rodrigues, O. M. P. R. (2013) Práticas educativas de mães de bebês: efeitos de um programa de intervenção. Psicologia em Estudo, 18(4) 599-609. https://doi.org/10.1590/S1413-73722013000400003

Nunes, S. A. N., Faraco, A.M.X., Vieira, M. L., Lisboa, C. S. M., Rubin, K.H. (2015). Relação entre Práticas Parentais e Problemas de Externalização e Internalização: Papel Mediador do Vínculo do Apego. Interação em Psicologia, Curitiba, 19(3), 371-383. http://dx.doi.org/10.5380/psi.v19i3.32371
Pestana, M. H., \& Cageiro, J. N. (2014). Análise de dados para Ciências Sociais: a complementaridade do SPSS. ( $6^{\mathrm{a}}$ ed.). Lisboa: Edições Sílabo.

Ramos, S. H. A. S., \& Furtado, E. F. (2007). Depressão puerperal e interação mãe-bebê: um estudo piloto. Psicologia em Pesquisa, 1(1), 20-28.

Rodrigues, O. M. P. R., \& Nogueira, S. C. (2016). Práticas Educativas e Indicadores de Ansiedade, Depressão e Estresse Maternos. Psicologia: Teoria e Pesquisa, 32(1), 35-44. https://doi.org/10.1590/0102-37722016012293035044

Rodrigues, O. M. P. R., Altafim, E. R. P., \& Schiavo, R. de A. (2011). Práticas parentais de mães adultas e adolescentes com bebês de um a doze meses. Aletheia, 34, 96-108.

Rodrigues, O. M. P. R., Nogueira, S. C., \& Altafim, E. R. P. (2013). Práticas parentais maternas e a influência de variáveis familiares e do bebê. Pensando Famílias, 17(2), 71-83.

Sameroff, A. (2009). The transactional model of development: How children and contexts shape each other. American Psychological Association.

Sampaio, I. T. A., \& Gomide, P. I. C. (2007). Inventário de Estilos Parentais (IEP) - Gomide (2006) - Percurso de Padronização e Normatização. Psicologia Argumento, 25(48), 15. https://doi.org/10.7213/rpa.v25i48.19675

Seixas, Í., Barbosa, M., \& Fuertes, M. (2017). Contributos para a auto-regulação do bebé no Paradigma Face-to-Face Still-Face. Análise Psicológica, 35(4), 469-485. https://dx.doi. org/10.14417/ap.1280

Servilha, B., \& Bussab, V. S. R. (2015). Interação Mãe-Criança e Desenvolvimento da Linguagem: A Influência da Depressão Pós-Parto. Psico (PUCRS), 46(1), 101-109. https://doi. org/10.15448/1980-8623.2015.1.17119

Simões, S. C. C. (2011). Influência dos estilos educativos parentais na qualidade da vinculação de crianças em idade escolar em diferentes tipos de família. [Tese de Doutorado, Universidade do Porto]. Repositório Aberto da Universidade do Porto. https://hdl.handle.net/10216/56783

Tronick, E., Als, H., Adamson, L., Wise, S., \& Brazelton, T. B. (1978). The Infant's Response to Entrapment between Contradictory Messages in Face-to-Face Interaction. Journal of the American Academy of Child Psychiatry, 17(1), 1-13. https://doi.org/10.1016/S0002-7138(09)62273-1

Varão, A. S. (2012). Um jogo a dois: Interação mãe-bebé e auto-regulação infantil. [Dissertação de Mestrado, Instituto Politécnico de Lisboa]. Repositório Científico do Instituto Politécnico de Lisboa. http://hdl.handle.net/10400.21/1979

Data de submissão: $11 / 09 / 2020$ Primeira decisão editorial: 16/12/2020 Aceite em: 29/01/2021 